\title{
Guest editorial: Event-based video analysis/retrieval
}

\author{
Anastasios Doulamis • Nikolaos Doulamis • \\ Luc van Gool • Mark Nixon
}

Published online: 15 October 2013

(C) Springer Science+Business Media New York 2013

Identification of events from visual cues is in general an arduous task because of complex motion, cluttered backgrounds, occlusions, and geometric and photometric variations of the physical objects. This is even more challenging in case of detection of a logical chain of events, i.e., of a sequence of events called a workflow, and in case of the presence of multiple workflows of events in the environment, able to interact one with the other and affect one the outcome of the other.

The recent research advances in computer vision and pattern recognition society have stimulated the development of a series of innovative algorithms, tools and methods for salient object detection and tracking in still images/video streams. These techniques are framed with appropriate descriptors (usually with invariance properties) such as the Scale-Invariant Feature Transform (SIFT) or the Speeded Up Robust Features (SURF), or the MPEG-7 visual descriptors. All these research methods can be considered as initial steps towards the ultimate goal for behavior/event understanding. However, automatic comprehension of someone's behavior within a scene or even automatic supervision of workflows (e.g., industrial processes) is a complex research field of great attention but with limited results so far.

Most of the current approaches presented involve machine learning theories, such as supervised or semi-supervised methods, object tracking algorithms, adaptation mechanisms to handle complex, dynamic and abrupt visual conditions and application-specific analysis topics.

On the other hand, during the past few years, more and more people have been coping with the so called "Information Overload" phenomenon. On the basis of the i) diversity and plenitude of media information currently available on the web and ii) the gradual but quick role shift of the users from being solely content consumers to acting both as content consumers

\footnotetext{
A. Doulamis $(\bowtie)$

Technical University of Crete, Kounoupidiana, Chania, Grete, Greece e-mail: adoulam@ergasya.tuc.gr

N. Doulamis

National Technical University of Athens, Zografou, Athens, Greece e-mail: ndoulam@cs.ntua.gr

L. van Gool

Eidgenoessische Technische Hochschule Zuerich (ETHZ), Zuerich, Switzerland e-mail: vangool@vision.ee.ethz.ch
}

M. Nixon

University of Southampton, Southampton, UK

e-mail:msn@ecs.soton.ac.uk 
and content providers a new challenge is emerging: how to make the most out of these vast amounts of media information quickly searchable often under a personalized manner.

This issue of Multimedia Tools and Applications Journal (MTAP) covers these two research challenging issues in computer vision and multimedia. The issue is mainly focused on visual events analysis and understanding with applications on visual retrieval topics. The call was published in MTAP journal. In parallel the Guest Editors disseminated the event in several groups and societies researching on this topic.

The call attracted many submissions all over the world, from Europe, America (mainly from USA and Canada), Australia, Asia (mainly from China, and Japan). All articles underwent a peer review process for at least three reviewers, while most of them received four or even five reviews. All non-rejected articles underwent revision to meet the reviewers' and editors' comments, while some of them were sent for a second or even a third revision at least all comments were carefully addressed. Failure to meet these tough conditions led to rejection.

After completion of this hard process only eight papers were finally accepted for publication in MTAP, while most of the submitted ones were rejected. The accepted papers cover most of the research components needed for action recognition and event detection from visual data. In particular, the papers of this issue concern (i) the use and/or fusion of new features able to improve the event detection performance, (ii) visual event analysis under complex conditions such as the industrial ones, (iii) environmental event detection on exploiting depth sensors cameras, (iv) extraction of song meanings from movies and finally (v) multimedia content search on the use of crowd-sourcing information and social awareness.

In particular, the work of Benmokhtar et al. proposes a new feature fusion framework able to analyzing and detecting visual events. Another approach is given in the article entitled "Efficient tracking using a robust motion estimation technique". Instead of fusing visual features, the authors of this article introduce a new motion feature able to track salient objects within complex visual scenes while leaving the non-salient ones intact. The work entitled "A top-down event-driven approach for concurrent activity recognition" proposes a top-down algorithm for identifying complex industrial workflows from a set of visual cameras. Great research challenges are imposed even from the content of the sequences used. In addition, a feedback mechanism is imposed to improve event detection analysis under such environments. Verstockt et al. proposes a scheme for detecting fires on the use of depth sensors like multimodal Time of Flight.

The remaining four articles are focused more on retrieving visual content from large datasets. Content retrieval exploits visual analysis on salient actions taking place within imagery data. A connecting link among classical event detection algorithms and the ones proposed for visual retrieval can be considered the article "Event-driven video adaptation: A powerful tool for industrial video supervision”. In this work, Doulamis et. al. proposes a new video adaptation scheme able to update visual content with respect to users' needs and contextual preferences in terms of network capacity, location, terminal devices properties and accessibility.

The work of "Mining movie archives for song sequences" mines songs from movies' archives. On the other hand, Chorianopoulos et al. propose in "VideoSkip: event detection in social web videos with an implicit user heuristic" an event-based social web interface for videos. Finally the work entitled "automatic annotation of image databases based on implicit crowdsourcing, visual concept modeling and evolution" introduces new methods for annotating large video databases by exploiting crowdsourcing information on users that implicitly evaluate the visual content.

The Guest Editors express their thanks to the Editor-in-Chief of Multimedia Tools and Applications (MTAP) journal, Prof. Borko Furht for supporting this call. The Guest Editors would also like to give special thanks to all the anonymous Reviewers for their excellent work in reading and judging on the quality of the papers. 


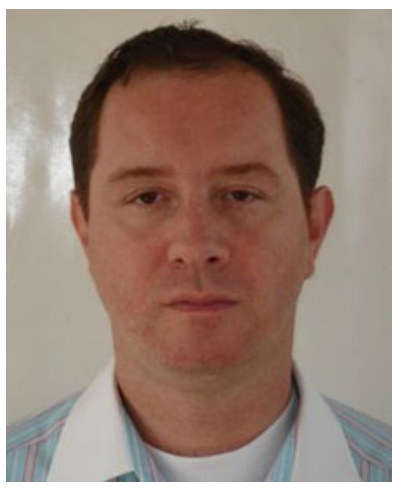

Anastasios Doulamis received the Diploma degree in Electrical and Computer Engineering from the National Technical University of Athens (NTUA) in 1995 with the highest honor (9.52 out of 10, first ranked among all classmates) and the $\mathrm{PhD}$ degree in Electrical and Computer Engineering from NTUA in 2000. He is currently Associate Professor in Technical University of Crete, while he has also elected in the faculty of the National Technical University of Athens. Prof. Anastasios Doulamis has received several awards and prizes during his studies, including the Best Greek Student in all fields of engineering in national level in 1995, the Best Graduate Thesis Award in the area of Electrical Engineering in 1996 and several prizes from the National Technical University of Athens, the National Scholarship Foundation and the Technical Chamber of Greece. In 1997, he was given the NTUA Medal as Best Young Engineer. In 2000, he received the best Phd thesis award by the Thomaidion Foundation. He is author of more 150 papers in the area of multimedia processing and artificial intelligence among them more than 15 in IEEE/ACM journals papers and more than 43 journal papers. Prof. Anastasios Doulamis is the Coordinator of the EU funded project 4D Ch World, and the scientific responsible of the EU funded projects Poseidon, e-Park, ITN-DCH.

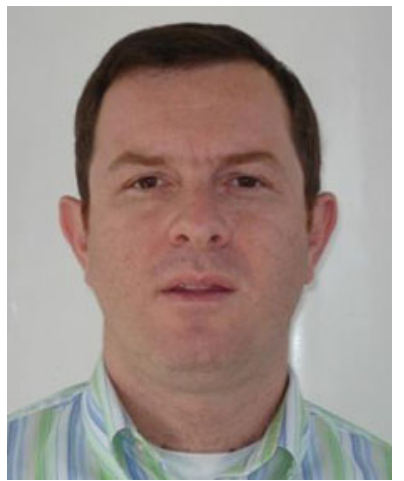

Nikolaos Doulamis received the Diploma degree in Electrical and Computer Engineering from the National Technical University of Athens (NTUA) in 1995 with the highest honor and the PhD degree in electrical and computer engineering from NTUA in 2000. He joined the Image, Video and Multimedia Lab of NTUA in 1996 as research assistant. His PhD thesis was supported by the Bodosakis Foundation Scholarship. Since 2005, he was serving as visiting professor at the National Technical University of Athens. In 2010, he was elected as Assistant Professor of the National Technical University of Athens.

Dr. Doulamis was awarded as the Best Greek Student in the field of engineering in national level by the Technical chamber of Greece in 1995. In 1996, he was received the Best Graduate Thesis Award in the area of electrical engineering. During his studies he has also received several prizes and awards from the National Technical University of Athens, the National Scholarship Foundation and the Technical Chamber of Greece. In 1997, he was given the NTUA Medal as Best Young Engineer. He has also served as program committee in several international conferences and workshops. He was given the Thomaidion Foundation best journal paper 
award. He is reviewer of IEEE journals and conferences as well as and other leading international journals. His research interest includes video transmission, content-based image retrieval, summarization of video sequences and intelligent techniques for video processing. Prof. Nikolaos Doulamis is the scientific responsible of the EU funded projects ITN-DCH, eVacuate, ZoneSec, Inacgus, VIMSEN.

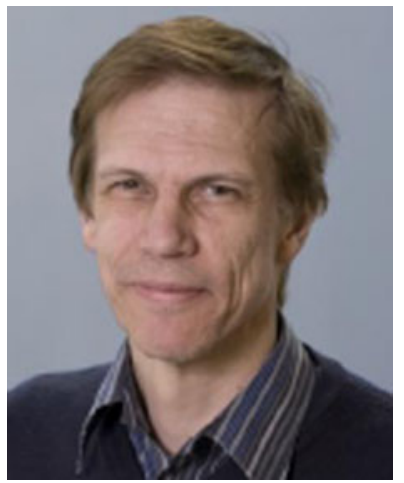

Luc Van Gool is both a full professor at Katholieke Universiteit Leuven (Belgium) and at the Eidgenössische Technische Hochschule (ETH) Zürich (Switzerland). He has worked on a broad variety of computer vision topics. His experience includes texture analysis and synthesis, 2D and 3D object and object class recognition, multi-view and structured light 3D reconstruction, visual robot navigation, tracking and motion analysis, and gesture and emotion interpretation. Several prizes testify to the international appreciation for his work, e.g. a David Marr prize (best paper award) at ICCV98 and the U.V. Helava Award, one of the most prestigious ISPRS awards in 2012. He was also awarded an ERC Advanced Grant in 2011 for his project VarCity (Variation and the City).

Luc Van Gool is initiator and editor-in-chief of Foundations and Trends in Computer Graphics and Vision. He is associate editor for IEEE T-PAMI and IJCV, the two main vision journals, for several years, as well as for MVA and ACM JOCCH. He was PC member and Area Chair for many major vision conferences and served as Program Chair of the Int. Conf. on Computer Vision (ICCV) 2005 in Beijing, and as General Chair for ICCV 2011 and the European Conf. on Computer Vision (ECCV) 2014, two of the 3 main computer vision conferences.

In 98 he co-founded the spin-off company Eyetronics (Belgium, US, Canada), specialised in 3D modelling. He was also the co-founder of the companies eSaturnus (medical multimedia solutions) (2006), kooaba (hyperlinking the real and digital world through object recognition on mobile phones) (2006), GeoAutomation (mobile mapping) (2007) and Procedural (procedural modeling of cities) (2007). His h-index is 71 , with a total of 21730 citations, according to scholar google. 


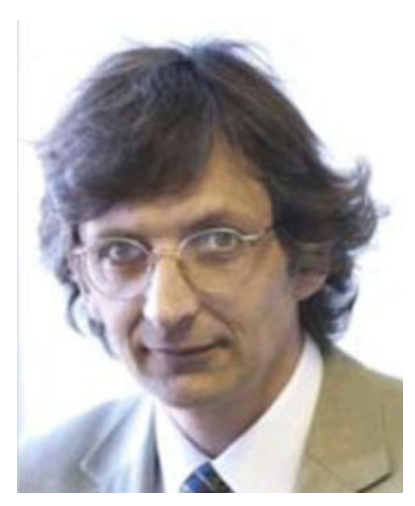

Mark Nixon is Professor in Computer Vision at the School of Electronics and Computer Science. His research interests are in image processing and computer vision including static and moving shape extraction (both parametric and non-parametric) which have found application in automatic face and automatic gait recognition and in medical image analysis. Amongst previous research contracts, he was Principal Investigator with John Carter on the DARPA supported project Automatic Gait Recognition for Human ID at a Distance, on the General Dynamics Defence Technology Centre's program on data fusion (biometrics, naturally), on the MoD/ARL (US) IBM-led Information Technology Alliance and we're currently working on the EU funded Tabula Rasa programme which is the first co-ordinated study of spoofing biometrics.

He was chaired of the 9th British Machine Vision Conference BMVC'98 held at Southampton in September '98 (an issue of Image and Vision Computing containing some of the most highly rated conference papers was published as Volume 18 Number 9). The BMVC'98 Electronic Conference Proceedings remain online via the British Machine Vision Association. Apart from being a programme member/ reviewer for other conferences, Josef Kittler and he chaired IAPR International Conference Audio Visual Biometric Person Authentication (AVBPA 2003) and was Publications Chair for the International Conference on Pattern Recognition (ICPR 2004) at Cambridge UK with Josef Kittler, he co-chaired the IEEE 7th International Conference on Face and Gesture Recognition FG2006 held at Southampton, UK in 2006. 